\title{
RADIO IMAGING OF THE SUPERNOVA REMNANT N132D IN THE LARGE MAGELLANIC CLOUD
}

\author{
JOHN R DICKEL \\ University of Illinois Astronomy Building \\ 1002 West Green Street \\ Urbana, IL 61801 \\ U.S.A.
}

\author{
D. K. MILNE \\ Australia Telescope National Facility* \\ P.O. Box 76 \\ Epping, N.S.W. 2121 \\ Australia
}

ABSTRACT. The first high resolution radio image of the supernova remnant N132D shows a complex shell structure.

As part of a program to investigate the detailed structure of supernova remnants (SNRs) in the Large Magellanic Cloud, the bright remnant N132D was mapped with the Australia Telescope. At a wavelength of $6 \mathrm{~cm}$ the half-power beamwidth is $8.3 \mathrm{x}$ 7.6 arcsec. The angular diameter of N132D is about 110 arcsec and the integrated flux density at $6 \mathrm{~cm}$ is $1.6 \mathrm{Jy}$. The $1 \mathrm{GHz}$-surface brightness of $1.5 \times 10^{-19} \mathrm{~W} \mathrm{~m}^{-2}$ $\mathrm{Hz}^{-1} \mathrm{sr}^{-1}$ is 5-10 times that of a typical SNR in the Milky Way with the same diameter of $33 \mathrm{pc}$. The image, after processing by maximum entropy techniques, is shown in Figure 1. The map looks somewhat similar to that obtained at X-ray wavelength by the High Resolution Imager on the Einstein Observatory and in an optical [OIII] photograph (see Mathewson et al). One difference is the presence of a faint outer arc to the southeast on the radio image giving the remnant a more circular outline than seen at the other wavelengths. There appear to be some real differences in the distributions of the magnetic fields plus relativistic particles responsible for the radio synchrotron emission and the hot, shocked gas responsible for the X-ray emission. N132D is also visible at a wavelength of $3 \mathrm{~cm}$ and those results will be forthcoming.

Reference. Mathewson, D.S., Ford, V.L., Dopita, M.A., Tuohy, I.R., Long, K.S and Helfand, D.J. 1983, Astrophys. J. Suppl. 51, 345

* Operated in association with the Division of Radiophysics by CSIRO

(c) 1991 IAU. Printed in the Netherlands. 


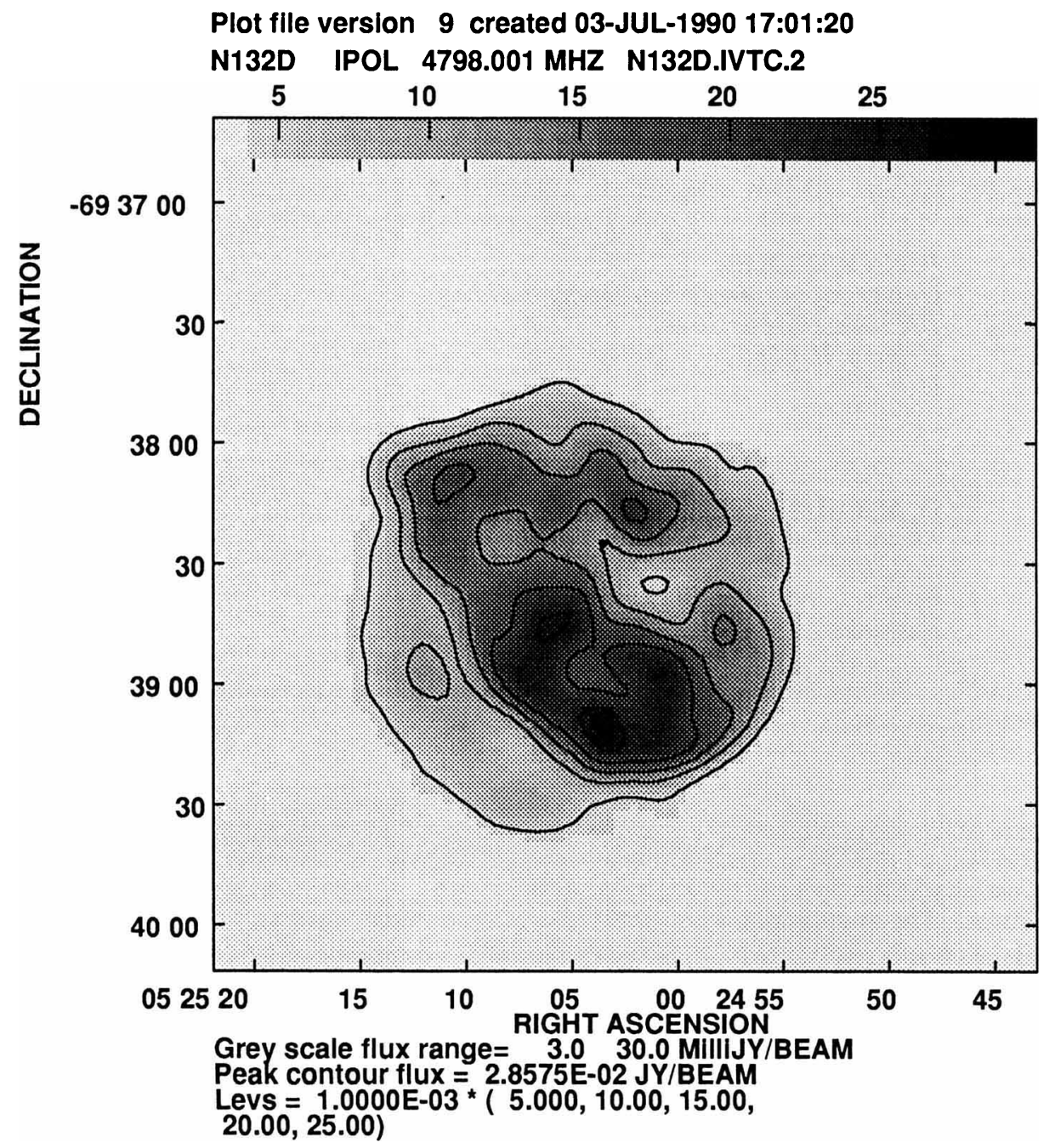

